International Journal of Wireless \& Mobile Networks (IJWMN) Vol. 4, No. 6, December 2012

\title{
COMBINING HARMONY SEARCH AND LEARNING AUTOMATA FOR TOPOLOGY CONTROL IN WIRELESS SENSOR NETWORKS
}

\author{
Mohamad Nikravan ${ }^{1}$ and Seyed Mahdi Jameii ${ }^{2}$ \\ ${ }^{1}$ Department of Computer Engineering, Shahr-e-Qods Branch, Islamic Azad niversity, \\ Tehran, Iran \\ Nikravan@Qodsiau.ac.ir \\ ${ }^{2}$ Department of Computer Engineering Shahr-e-Qods Branch, Islamic Azad University, \\ Tehran, Iran \\ Jamei@Qodsiau.ac.ir
}

\begin{abstract}
One of the critical challenges in wireless sensor networks (WSNs) is energy conservation. Topology control is a vital process to minimize energy consumption and maximize the network lifetime. In this paper, we propose a distributed topology protocol with transmission power adjustment based on harmony search and learning automata algorithms called HSLATC (Harmony Search and Learning Automata based Topology Control protocol). In HSLATC, proper transition radius of the sensor nodes can be determined. Because of the intelligent determining the transition radiuses of the nodes in the proposed protocol, it is able to provide the full connectivity in sparse deployment and decreases the energy consumption of the sensor network and prolongs the network lifetime. The simulation results show high efficiency of the proposed protocol.
\end{abstract}

\section{KEYWORDS}

Wireless Sensor Network, Topology Control, Network Lifetime, Harmony Search, Learning Automata.

\section{INTRODUCTION}

Wireless sensor network (WSN) is a self-organized network that consists of a large number of sensor nodes deployed on the ground, in the air, in vehicles, on bodies, under water and inside buildings. This kind of networks has become an emerging technology that has a wide range of potential applications including environment monitoring, object tracking, scientific observing and forecasting, traffic control and etc [1]. Each sensor node is a tiny device that includes three basic units: a sensing unit for data acquisition from the physical environment, a processing unit for local data processing and storage, and a wireless communication unit for data transmission to sink node or base station. However, sensor nodes are typically equipped with limited energy supplies (a battery), and it is impossible or inconvenient to recharge the batteries because nodes may be deployed in a hostile or unpractical environment [2] [3]. It has been demonstrated that both powering off redundant nodes and lowering radio power while maintaining node connections can contribute to efficient power saving [4].

In this paper, we focus on the optimal transmission power of nodes and the optimal network topology of WSNs. The goal of our research is to maximize the network lifetime. We propose a scheme of deciding the optimal transmission power for each node in WSNs called HSLATC ${ }^{1}$ and the scheme must ensure the connectivity of the network. The remaining of this paper is organized as follows: In Section 2 we present the related work in the context of topology control in WSNs. briefly. We have an overview on the Harmony Search (HS) algorithm in Section 3 and Learning Automata (LA) in section 4. Section 5 describes the proposed algorithm. Section 6

\footnotetext{
${ }^{1}$ Harmony Search and Learning Automata based Topology Control Protocol 
International Journal of Wireless \& Mobile Networks (IJWMN) Vol. 4, No. 6, December 2012

presents some simulation results and evaluates the algorithm for different configuration of sensor field. The paper concludes with Section 7.

\section{RELATED WORKS}

One major problem in the area of wireless sensor network is the topology control. Topology control protocols in WSNs construct an optimized network topology structure to satisfy the application requirements, such as network connectivity and coverage [5]. Most works on topology control are based on adjustable transmission power control and mainly focus on maintaining a connected topology while minimizing energy consumption of nodes to extend the lifetime of network [6]. In recent years, the researchers have already done a large amount of research work on power control for WSNs, and a lot of power control mechanisms have been proposed which try to design simple and practical protocols that build and maintain a reasonably good topology. So far, many protocols have been introduced for topology control in sensor networks. Topology control protocols are divided into homogeneous and heterogeneous. In homogeneous topology control, all network nodes use the same transition radius and topology control problem is to find a minimum value for transition radius considering the network characteristic such as network connectivity and coverage. While in heterogeneous control topology, network nodes can have non uniform transition radius [7]. $\mathrm{LMST}^{2}$ [8] and XTC ${ }^{3}$ [9] protocols are protocols of this group. A simple solution called COMPOW [10] and its basic idea is to apply a uniform transmission power to all sensor nodes and minimize the power without decreasing network connectivity. When nodes are uniformly deployed, COMPOW can have a good performance but when nodes are not uniformly deployed, the efficiency of COMPOW decreases, because some nodes will lead all nodes to use large transmission range, which results in more energy consumption. RAA- $2 \mathrm{~L}^{4}$ is another topology control protocol. In this protocol, each node chooses one of two transition radius $R_{S}$ or $R_{W}\left(R_{W}<R_{S}\right)$ [11]. If a node with transition radius of $R_{W}$ could communicate with all neighbors in transition radius $R_{S}$, it chooses transition radius $R_{W}$, else it chooses transition radius $R_{S}$. In RAA-3L ${ }^{5}$, each node chooses one of three transition radius: $R_{t}, R_{S}$ or $R_{W}\left(R_{W}<R_{t}<R_{S}\right)$.

Research [12] proposes the Optimal Geographical Density Control (OGDC) algorithm that addresses both sensing coverage and connectivity in wireless sensor networks. Their algorithm aims at computing the minimum number of nodes that must be kept awake such that both sensing coverage and connectivity are maintained. The algorithm is decentralized but requires the network to be sufficiently dense to guarantee connectivity.

Authors in [13] proposed LMA and LMN algorithms based on nodes degree. The basic idea is giving the upper limit and lower limit of nodes degree, every node adjust transmission power, and each node degree falls between the upper limit and lower limit. However the algorithms cannot guarantee the network connectivity. Li proposed algorithms based on proximity graph theory, Directed Relative Neighbourhood Graph (DRNG) and Directed Local Minimum Spanning Tree (DLMST) [14]. Nodes use maximum transmission power to form the topology graph $G$, find proximity graph $G^{\prime}$ according to some criterion, each node decides its transmission power in order to communicate with the farthest neighbourhood. Cerpa proposed an Adaptive Self-Configuring Sensor Networks Topologies (ASCENT) to maintain a data delivery [15]. In ASCENT, each node assesses its connectivity and adapts its participation in multi-hop network topology based on measured operating region. ASCENT neither can ensure network connectivity nor uniform energy consumption. Ye developed a protocol called Probing Environment and Adaptive Sensing (PEAS) [16]. In PEAS, each node has three statuses: sleeping, probing and working. Firstly nodes are in the status of sleeping, when sleeping timer overflow, nodes enter into working status, and broadcast detecting message as a certain transmission radius, if a node receives acknowledge, it will enter into sleeping status, otherwise

\footnotetext{
${ }^{2}$ Local Minimum Spanning Tree

${ }^{3}$ Extreme Topology Control

${ }^{4}$ Radius Adaptation Algorithm_2 Level

${ }^{5}$ Radius Adaptation Algorithm_3 Level
} 
International Journal of Wireless \& Mobile Networks (IJWMN) Vol. 4, No. 6, December 2012

it will enter into probing status until energy deplete. PEAS can provides asymptotic connectivity. Wang et al. proposed Coverage Configuration Protocol (CCP) [17], the basic idea is maximizing the number of sleeping nodes under the condition of $\mathrm{K}$-coverage and $\mathrm{K}$ connectivity. When transmission radius is two times of sensing range, if network K-coverage a bulge region, then the network must be K-connectivity. CCP can ensure the network connectivity through transmission range.

\section{HaRmony SEarch Algorithm}

HS algorithm, originated by Geem et al. [18], is based on natural musical performance processes that occur when a musician searches for a better state of harmony. HS has several advantages with respect to traditional optimization techniques such as the following [19]:

- HS imposes fewer mathematical requirements.

- HS is free from divergence.

- HS does not require initial value settings of the decision variables, thus it may escape the local optima. Furthermore it may be easily adapted for multi-modal problems.

- As the HS uses stochastic random searches, derivative information is unnecessary. HS has novel stochastic derivative.

- HS can handle both discrete and continuous variables.

- HS algorithm could overcome the drawback of genetic algorithm building block theory by considering the relationship among decision variables using its ensemble operation. HS generates a new vector, after considering all of the existing vectors, whereas the genetic algorithm only considers the two parent vectors.

The HS algorithm optimization procedure consists of the following five steps [20]:

Step 1: Problem and algorithm parameter initialization

Step 2: Harmony memory initialization and evaluation

Step 3: New harmony improvisation

Step 4: Harmony memory update

Step 5: Termination criterion check

\section{Learning Automat}

The automata approach to learning involves determination of an optimal action from a set of allowable actions. Learning Automata is an abstract model which randomly selects one action out of its finite set of action and performs it on a random environment. Environment then evaluates the selected action and responds to the automata with a reinforcement signal. Based on selected action and received signal, the automata updates its internal state and selects its next action. Figure1 depicts the relationship between an automaton and its environment.

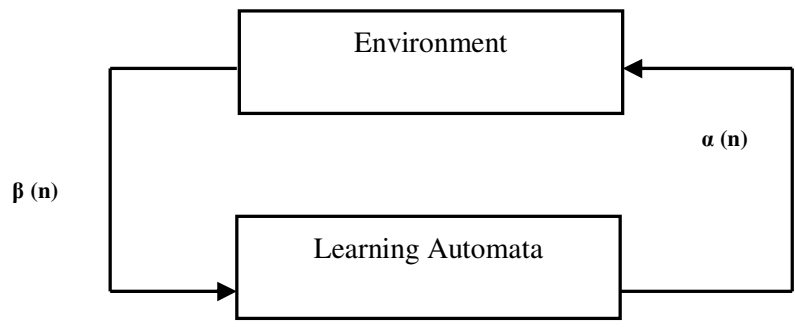

Figure 1. Relationship between Learning Automaton and its environment 
International Journal of Wireless \& Mobile Networks (IJWMN) Vol. 4, No. 6, December 2012

Environment can be defined by the triple $\mathrm{E}=\{\alpha, \beta, \mathrm{c}\}$, where $\alpha=\left\{\alpha_{1}, \alpha_{2}, \ldots, \alpha_{\mathrm{r}}\right\}$ represents a finite input set, $\beta=\left\{\beta_{1}, \beta_{2}, \ldots, \beta_{\mathrm{r}}\right\}$ represent the output set, and $\mathrm{c}=\left\{\mathrm{c}_{1}, \mathrm{c}_{2}, \ldots, \mathrm{c}_{\mathrm{r}}\right\}$ is set of penalty probabilities, where each element $c_{i}$ of c corresponds to one input of action $\alpha_{i}$. An environment in which $\beta$ can takes only binary value 0 or 1 is referred to as P-model environment. A further generalization of the environment allows finite output sets with more than two elements that take values in the interval [0-1]. Such an environment is referred to as Q-model. Finally, when the output of the environment is a continuous random variable which assumes values in the interval $[0,1]$, it is referred to as S-model. Learning automata are classified into fixed-structure and variable-structure. A fixed structure learning automaton is represented by a quintuple $\left\langle\alpha, \varphi, \beta, F, G>\right.$ where: $\alpha=\left\{\alpha_{1}, \alpha_{2}, \ldots, \alpha_{r}\right\}$ is set of actions that it must choose from. $\Phi=\left\{\Phi_{1}, \Phi_{2}, \ldots, \Phi_{s}\right\}$ is the set of internal states. $\beta=\{0,1\}$ is the set of inputs where 1 represents a penalty and 0 represent a reward. F: $\Phi \times \beta \rightarrow \Phi$ is a map called the state transition map. It defines the transition of the state of the automaton on receiving an input.

$\mathrm{G}: \Phi \rightarrow \alpha$ is the output map and determines the action taken by the automaton if it is in state $\Phi_{\mathrm{j}}$. A variable-structure learning automaton is defined by the quadruple $\{\alpha, \beta, \mathrm{P}, \mathrm{T}\}$ in which $\alpha=\left\{\alpha_{1}, \alpha_{2}, \ldots, \alpha_{\mathrm{r}}\right\}$ represents the action set of the automaton, $\beta=\left\{\beta_{1}, \beta_{2}, \ldots, \beta_{\mathrm{r}}\right\}$ represent the input set, and $\mathrm{P}=\left\{\mathrm{P}_{1}, \mathrm{P}_{2}, \ldots, \mathrm{P}_{\mathrm{r}}\right\}$ represent the action probability set, and finally $\mathrm{P}(\mathrm{n}+1)=\mathrm{T}[\alpha(\mathrm{n}), \beta(\mathrm{n}), \mathrm{P}(\mathrm{n})]$ represent the learning algorithm. This automaton operates as follows. Based on the action probability set $\mathrm{P}$, automata randomly selects an action $\alpha_{\mathrm{i}}$, and performs it on the environment. After receiving the environment's reinforcement signal, automaton updates its action probability set based on (1) for favorable responses, and based on (2) for unfavorable ones.

$$
\begin{array}{ll}
\mathrm{P}_{\mathrm{i}}(\mathrm{n}+1)=\mathrm{p}_{\mathrm{i}}(\mathrm{n})+\mathrm{a} \cdot\left(1-\mathrm{p}_{\mathrm{i}}(\mathrm{n})\right) & \forall \mathrm{j} \mathrm{j} \neq \mathrm{i} \\
\mathrm{P}_{\mathrm{j}}(\mathrm{n}+1)=\mathrm{p}_{\mathrm{j}}(\mathrm{n})-\mathrm{a} \cdot \mathrm{p}_{\mathrm{j}}(\mathrm{n}) & \\
\mathrm{P}_{\mathrm{i}}(\mathrm{n}+1)=(1-\mathrm{b}) \cdot \mathrm{p}_{\mathrm{i}}(\mathrm{n}) & \\
\mathrm{P}_{\mathrm{j}}(\mathrm{n}+1)=\mathrm{b} /(\mathrm{r}-1)+(1-\mathrm{b}) \cdot \mathrm{p}_{\mathrm{j}}(\mathrm{n}) & \forall \mathrm{j} j \neq \mathrm{i}
\end{array}
$$

In these two equations, $\mathrm{a}$ and $\mathrm{b}$ are reward and penalty parameters, respectively. For $\mathrm{a}=\mathrm{b}$, learning algorithm is called $\mathrm{L}_{\mathrm{R}-\mathrm{P}}$, for $\mathrm{b}<<\mathrm{a}$, it called $\mathrm{L}_{\mathrm{R}} \mathcal{E}_{\mathrm{P}}$ and for $\mathrm{b}=0$, it is called $\mathrm{L}_{\mathrm{R}-\mathrm{I}}$. For more information the reader may refer to [21].

\section{Proposed Protocol}

In this section, we propose a topology control solution based on harmony search algorithm and learning automata and try to decrease the average of transition radius of nodes without decreasing the connectivity of the sensor network. At the beginning of this section, the assumption made in the proposed protocol will be descried:

We assume that each sensor node has adjustable transition radius which can be between a minimum and a maximum transition radius:

- $R_{\text {min }}$ : transition radius with minimum power

- $R_{\text {max }}$ : transition radius with maximum power

- $R_{T}$ : selective transition radius of node

The value of $R_{T}$ should be between the $R_{\text {min }}$ and $R_{\max }\left(R_{\min } \leq R_{T} \leq R_{\max }\right)$. Value of transition radius $R_{\min }$ and $R_{\max }$ will be calculated based on $R_{t}$. Value of transition radius $R_{t}$ is determined proportional to the network density [22].

Assume that the sensor set is $S=\left\{n_{1}, n_{2}, \ldots, n_{\mathrm{N}}\right\}$ and the transition radius set is $R_{\mathrm{T}}=\left\{R_{1}, R_{2}, \ldots, R_{N}\right\}$, where $R_{i}$ is the transition radius of node $n_{i}$, and $R_{i} \in\left[R_{\text {min }}, R_{\text {max }}\right]$. 
International Journal of Wireless \& Mobile Networks (IJWMN) Vol. 4, No. 6, December 2012

Similar to the cluster-based coverage control scheme introduced in [2], we use a cluster-based topology control scheme in this paper which is scheduled into rounds. In each round, firstly, the target area is divided into several equal squares. Then the node in each square having the largest energy will be chosen as the cluster-head, and the procedure of selecting the cluster-head is the same work in [23]. Each cluster-head is equipped with one learning automata and will choose transition radiuses of its member nodes. In the next round, another sensor set will be selected as cluster heads. So, the energy consumption among all the sensor nodes can be balanced well.

The energy consumed by a sensor node to deal with a transmission task is proportional to $\mathrm{R}_{\mathrm{T}}{ }^{2}$ or

$\mathrm{R}_{\mathrm{T}}{ }^{4}$, where $\mathrm{R}_{\mathrm{T}}$ is the transition radius of node [24]. In this paper, we take the energy onsumption of the transition task as $\alpha \cdot \mathrm{R}_{\mathrm{T}}{ }^{2}$, where $\alpha$ is the factor. Also, for the brief of the energy consumption analysis, we only consider the energy consumed by the transmission function, and do not include the energy consumption of calculation and sensing [2]. Thus, the energy consumption of the sensor set, which is related to the sum of the sensor's transition radius squared, is defined as (3):

$$
E_{\text {total }}=\alpha \cdot \sum_{\mathrm{i}=1 \text { to N }} R_{\mathrm{i}}{ }^{2}
$$

In the proposed protocol, at first, a primary population of transition radius set is selected randomly. Each transition radius set represents one configuration of sensor network. We consider the mentioned population as harmony memory. Then we try to improve the harmony memory by creating a new transition radius set. If the new transition radius set is better than the worst transition radius set existing in harmony memory, the worst one will be replaced with it. The process of providing new transition radius set and replacing the worst transition radius set with the new one continue until meeting termination criteria. Algorithm continues until achieving a certain number of iterations. Finally, the best transition radius set existing in the harmony memory is selected as the response. So, the proposed algorithm is presented briefly in some steps as follows:

Phase1. Initialization:

Step1: Initialize the problem and algorithm parameters.

Step2: Initialize the harmony memory with the transition radius sets randomly.

Phase2. Repeating main loop of algorithm until meeting termination criteria:

Step3: Improvise a new transition radius set.

Step4: Update the harmony memory.

Step5: Check the stopping criterion.

These steps are described in the next five subsections.

\section{A. Step1. Initialize the problem and algorithm parameters}

In this step, the optimization problem is specified as (4):

Maximize $f\left(R_{T}\right)=\operatorname{Con}\left(R_{T}\right) \times\left(1 /\left(E_{\text {total }}\left(R_{T}\right)+\varepsilon_{1}\right)\right)$

In (4), the amount of function $\operatorname{Con}\left(R_{T}\right)$ is calculated according to (5):

$$
\operatorname{Con}\left(\mathrm{R}_{\mathrm{T}}\right)= \begin{cases}1 & \text { If } R_{T} \text { provides the full connectivity } \\ \varepsilon_{2} & \text { Else }\end{cases}
$$

Where $R_{T}$ is the set of transition radius; $N$ is the number of nodes, $R_{i}$ is the transition radius of node $n_{i}$, that is $R_{\min } \leq R_{i} \leq R_{\max } . R_{\min }$ and $R_{\max }$ are the lower and upper bounds for transition radius of each node. Also $\varepsilon_{1}$ and $\varepsilon_{2}$ shows two very small positive numbers that should be selected properly such a way that $f\left(R_{T}\right)$ function value doesn't exceed the threshold value. 
International Journal of Wireless \& Mobile Networks (IJWMN) Vol. 4, No. 6, December 2012

As mentioned before, $E_{\text {total }}\left(R_{T}\right)$ is the transition energy consumption of the sensor network based on (3), so:

If $\varepsilon_{2}=1 \Rightarrow f\left(\mathrm{R}_{\mathrm{T}}\right)=\left(1 /\left(E_{\text {total }}\left(R_{T}\right)+\varepsilon_{1}\right)\right) \Rightarrow$

Maximum $f\left(R_{T}\right)=f\left(R_{\mathrm{MIN}}\right), R_{\mathrm{MIN}}=\left(R_{\min }, R_{\min }, \ldots R_{\text {min }}\right)$.

Therefore, according to $(6), f\left(R_{T}\right)$ function value is in inverse ratio to the transition energy consumption:

Also, the HS algorithm parameters are also initialized in this step:

- HMS: The harmony memory size (or the number of the transition radius sets in the harmony memory.

- HMCR: The harmony memory considering rate.

- PAR: pitch adjusting rate.

- NI: The number of improvisations, or stopping criterion.

HMCR and PAR are two parameters that are used to improve the transition radius sets. Both are defined in Step 3 (i.e. improvise a transition radius set).

B. Step2.Initialize the harmony memory

The Harmony Memory (HM) is a memory location where all transition radius sets are stored. In this step, we consider a Harmony Memory consists of one HMS group of the transition radius sets according to Figure 2 . Each the transition radius set, $R_{T}^{i}=\left(R_{1}{ }^{i}, R_{2}{ }^{i}, R_{3}{ }^{i}, \ldots, R_{N}{ }^{i}\right)$, represents one configuration of sensor network such a way that the transition radius of each $\mathrm{j}^{\text {th }}$ node equals to $R_{j}^{i}$. The fitness value of this configuration is shown by $f\left(R_{T}^{i}\right)$.

$$
\mathrm{HM}=\left[\begin{array}{lllll|l}
R_{1}^{1} & R_{2}^{1} & \cdots & R_{N-1}^{1} & R_{N}^{1} & f\left(R^{1}\right) \\
R_{1}^{2} & R_{2}^{2} & \cdots & R_{N-1}^{2} & R_{N}^{2} & f\left(R^{2}\right) \\
\vdots & \vdots & \vdots & \vdots & \vdots & \vdots \\
R_{1}^{H M S-1} & R_{2}^{H M S-1} & \ldots & R_{N-1}^{H M S-1} & R_{N}^{H M S-1} & f\left(R^{H M S-1}\right) \\
R_{1}^{H M S} & R_{2}^{H M S} & \cdots & R_{N-1}^{H M S} & R_{N}^{H M S} & f\left(R^{H M S}\right)
\end{array}\right]
$$

Figure 2. The Harmony Memory (HM) is a memory location where all transition radius sets are stored.

So, one N $\times$ HMS matrix of transition radii will be obtained. These transition radii are initialized randomly. The fitness value of each transition radius set $R_{T}^{i}$ is represented by $f\left(R_{T}^{i}\right)$ and it is calculated by (7):

$f\left(\mathrm{R}_{\mathrm{T}}^{\mathrm{i}}\right)=\operatorname{Con}\left(\mathrm{R}_{\mathrm{T}}^{\mathrm{i}}\right) \times\left(1 /\left(E_{\text {total }}\left(R_{T}^{\mathrm{i}}\right)+\varepsilon_{1}\right)\right)$

$R_{T}^{\mathrm{i}}=\left(R_{1}{ }^{i}, R_{2}{ }^{i}, R_{3}{ }^{i}, \ldots, R_{N}{ }^{i}\right), \mathrm{i}=1,2,3, \ldots, \mathrm{HMS}$

\section{Step3. Improvise a transition radius set}

Generating a new harmony (a new transition radius set) is called 'improvisation'. A new transition radius set, $R_{\mathrm{T}}^{\prime}=\left(R_{1}^{\prime}, R_{2}^{\prime}, R_{3}^{\prime}, \ldots, R_{\mathrm{N}}^{\prime}\right)$, is generated based on three rules:

- Memory consideration

- Random selection

- Pitch adjustment 
International Journal of Wireless \& Mobile Networks (IJWMN) Vol. 4, No. 6, December 2012

In the memory consideration, the value of the first transition radius $\left(R^{\prime}{ }_{1}\right)$ for the new transition radius set is chosen from any of the values in the specified HM range $\left(R_{1}^{\prime}{ }_{1}{ }, \mathrm{R}_{1}^{\prime}{ }_{1}, R_{1}^{\prime}{ }^{3}, \ldots, R_{1}^{\prime}{ }^{\mathrm{HMS}}\right)$. Values of the other transition radiuses $\left(R_{2}^{\prime}, R_{3}^{\prime}, \ldots, R_{\mathrm{N}}^{\prime}\right)$ are chosen in the same manner. The HMCR which varies between 0 and 1 , is the rate of choosing one value from the historical transition radius values stored in the HM, while $(1-\mathrm{HMCR})$ is the rate of randomly selecting one value from the possible range of values (i.e. one value between $\left.\left[R_{\min }, R_{\max }\right]\right)$. For example, a HMCR of 0.85 indicates that the HS algorithm will choose the transition radius value from historically stored values in the HM with the probability of $85 \%$ or from $\left[R_{\min }, R_{\max }\right]$ with the probability of (100-85)\%. The value of HMCR can be increased or decreased by the learning automata of cluster head which is doing the harmony search algorithm. Each learning automata can choose one of this two actions:

- Action1: selecting one value from the historical transition radius values stored in the HM with the probability of $\mathrm{P}_{1}=\mathrm{HMCR}$

- Action2: randomly selecting one value from the possible range of values with the probability of $\mathrm{P}_{2}=(1-\mathrm{HMCR})$

If choosing one of this actions result in improving the fitness function, the selected action will be rewarded according to (1), else it will be penalized according to (2). So, the learning automata will learn to choose the best value for HMCR.

Every transition radius $R_{\mathrm{i}}^{\prime} \in R_{T}^{\prime}=\left\{R_{1}^{\prime}, R_{2}^{\prime}, \ldots, R_{N}^{\prime}\right\}$ obtained by the memory consideration is examined to determine whether it should be pitch-adjusted. This operation uses the PAR parameter. The value of PAR sets the rate of doing pitch adjustment, while the value of (1-PAR) sets the rate of doing nothing. If pitch adjustment operator is applied, $R_{i}^{\prime}$ is replaced as (8):

$R_{i}^{\prime} \leftarrow R_{i}^{\prime} \pm \operatorname{rand}() \times\left(\left(R_{\max }-R_{\min }+1\right) \times \mu\right)$

Where rand() is a random number between 0 and 1 . Also, as already stated, $R_{\min }$ and $R_{\max }$ are the lower and upper bounds for transition radius of nodes. $\mu$ is a constant factor between 0 and 1 (e.g. $\mu=0.5$ ). In Step 3, HM consideration, pitch adjustment or random selection is applied to each variable of the new transition radius set in turn.

\section{Step4. Update harmony memory}

If the new transition radius set, $R_{T}^{\prime}=\left(R_{1}^{\prime}, R_{2}^{\prime}, R_{3}^{\prime}, \ldots, R_{N}^{\prime}\right)$, is better than the worst transition radius set existing in Harmony Memory, judged in terms of the objective function value $f\left(R_{T}\right)$, the worst one will be replaced with it.

\section{E. Step5. Check stopping criterion}

If the stopping criterion (i.e. maximum number of improvisations) is satisfied, computation is terminated. Otherwise, Steps 3 and 4 are repeated.

After ending the main loop of algorithm, the transition radius set with most objective function is selected among transition radius sets existing in Harmony Memory.

\section{Simulation Result}

In this section, our proposed protocol is simulated and compared to RAA-2L, RAA-3L [11] and homogeneous mode (HOM) using NS2 simulator. In the simulation, we assume a target area with a size of $150 \times 150 \mathrm{~m}^{2}$. We deploy the sensor nodes randomly in the target area. The number of nodes, $N$, in different configurations are considered as 200, 250, 300, 350, 400, 450, 500, 550 and 600 respectively. Each node has a transition range between $R_{\min }$ and $R_{\max }$. These transition ranges for different configurations is shown in Table 1. Other assumptions are shown in Table 2. 
Table 1. Parameters values

\begin{tabular}{|c|c|c|c|c|c|c|}
\hline NI & HMS & HMCR & PAR & $\boldsymbol{\varepsilon}_{\mathbf{1}}$ & $\boldsymbol{\varepsilon}_{\mathbf{2}}$ & $\boldsymbol{\varepsilon}_{\mathbf{3}}$ \\
\hline 300 & 50 & 0.85 & 0.02 & 1 & 0.001 & 0.01 \\
\hline
\end{tabular}

Table 2. Transition ranges for different configurations

\begin{tabular}{|c|c|c|c|}
\hline Number of nodes (N) & Rmin & Rt & Rmax \\
\hline 200 & 87 & 109 & 136 \\
\hline 250 & 75 & 94 & 118 \\
\hline 300 & 69 & 85 & 108 \\
\hline 350 & 64 & 80 & 100 \\
\hline 400 & 59 & 75 & 93 \\
\hline 450 & 56 & 70 & 88 \\
\hline 500 & 54 & 67 & 84 \\
\hline 550 & 50 & 63 & 79 \\
\hline 600 & 48 & 60 & 75 \\
\hline
\end{tabular}

In the first experiment, the average numbers of neighbors for HSLATC, RAA-2L, RAA-3L and HOM protocols are measured. The purpose of this experiment is to evaluate the ability of the proposed protocol to decrease the number of neighbors.

In the second experiment, we measured the average of transition radius of network for HSLATC, RAA-2L, RAA-3L and HOM protocols. In the third experiment, network connectivity in HSLATC is measured and compared to RAA-2L, RAA-3L, HOM and MAX-RANGE protocols. Note that, in MAX-RANGE, all of nodes have maximum transition radius.

The complete network connectivity means ability of communicating with all of network nodes. A WSN can be modeled as a graph $G=(V, E)$, where $V$ is the set of sensor nodes and $E$ is the set of wireless links. Thus, we define the probability of complete connectivity of the network, $P_{C}$, as (9):

$$
\begin{aligned}
& \mathrm{P}_{\mathrm{C}}=\Sigma_{\mathrm{i}=1 \text { to Nd }} \mathrm{C}_{\mathrm{i}} / \mathrm{N}_{\mathrm{d}} \\
& \mathrm{C}_{\mathrm{i}}= \begin{cases}1 & \text { if } \mathrm{MCP}=n \\
\varepsilon_{3} & \text { other }\end{cases}
\end{aligned}
$$

In (9), MCP is the biggest connected component of the network and $\mathrm{n}$ is the number of network nodes. Also $\varepsilon_{3}$ shows very small positive number and $N_{d}$ is the number of different configuration of network nodes. In this experiment, we suppose $\mathrm{N}_{\mathrm{d}}$ is equal to 50. The result of these simulations is depicted in figure 3, figure 4 and figure 5 . As can be seen, the average number of neighbors in the HSLATC is less than those of other protocols. Note that a lower number of neighbors results in lower interference between the nodes. Due to the proposed protocol accuracy in adjusting transition radius of the sensor nodes, it is able to provide the full connectivity in sparse deployment. Moreover, as the result of increasing nodes density, the proposed protocol decreases the average of transition radiuses of the network. As a result, the proposed protocol decreases the energy consumption of the sensor networks and prolongs the network lifetime. 
International Journal of Wireless \& Mobile Networks (IJWMN) Vol. 4, No. 6, December 2012

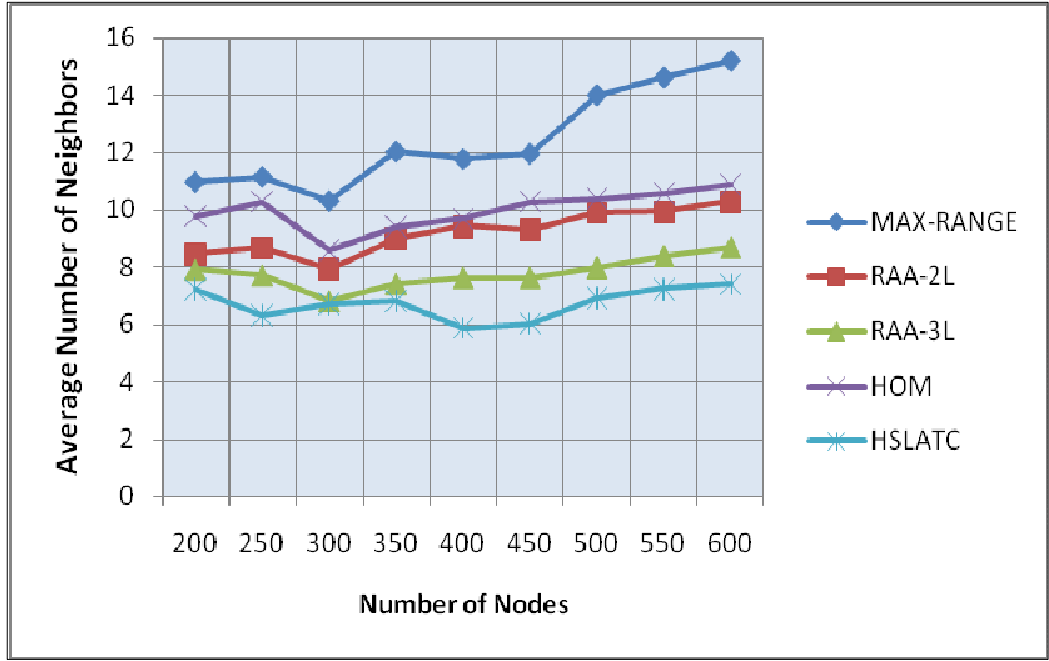

Figure 3. The average Number of Neighbors

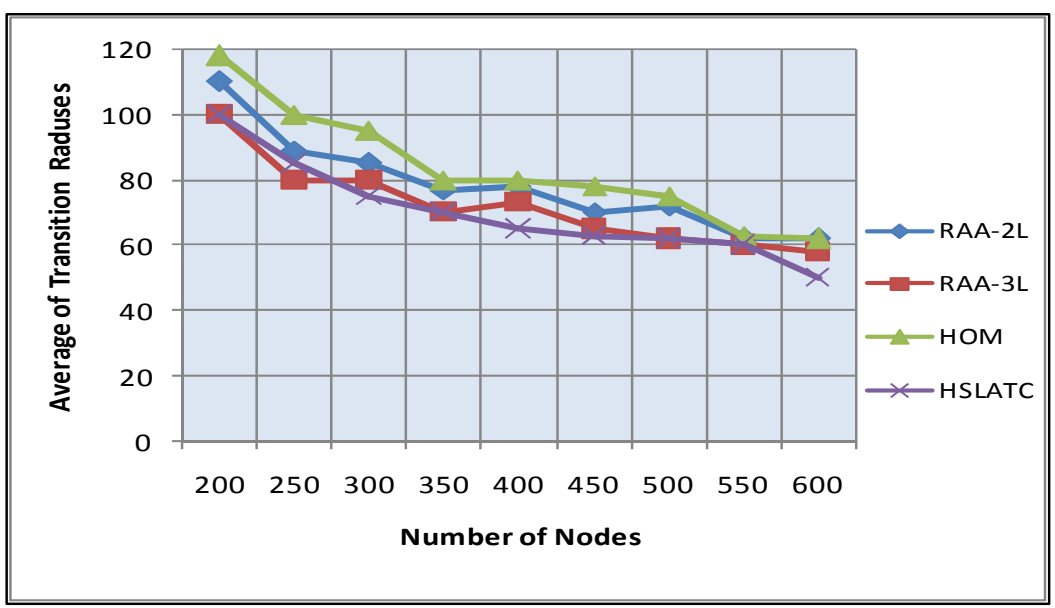

Figure 4. The average of transition radius of network

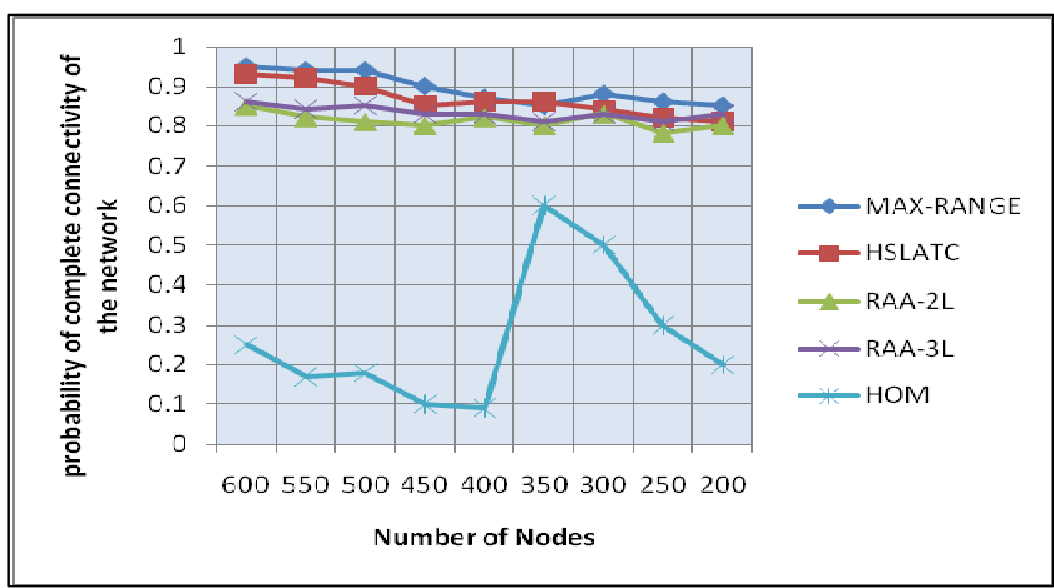

Figure 5. The probability of complete connectivity of the network 
International Journal of Wireless \& Mobile Networks (IJWMN) Vol. 4, No. 6, December 2012

\section{CONCLUSION}

In this paper we proposed a distributed topology protocol with transmission power adjustment based on harmony search and learning automata algorithms called HSLATC. In HSLATC, proper transition radius of the sensor nodes can be determined using the harmony search algorithm. Due to the proposed protocol accuracy in selecting the transition radius of sensor nodes, it is able to provide the full connectivity in sparse deployment. Moreover, as the result of increasing nodes density, the proposed protocol decreases the average of transition radius of network and prolongs the network lifetime. We have simulated our protocol and simulation results show high efficiency of the proposed protocol.

\section{ACKNOWLEDGMENTS}

This work is a part of the research project entitled "A Novel Method for Prolonging the Lifetime of Wireless Sensor Networks" that has been supported by the Islamic Azad University, Shahr-eQods branch.

\section{REFERENCES}

[1] Y. Li, C. Ai, Z. Cai, and R. Beyah, (2009 ) "Sensor scheduling for p-percent coverage in wireless sensor networks", Springer Science Business Media, LLC.

[2] J. Jia, J. Chena, G. Changa, Y. Wena, and J. Songa, (2009) "Multi-objective optimization for coverage control in wireless sensor network with adjustable sensing radius", Elsevier, Computers and Mathematics with Applications, Vol. 57, pp. 1767-1775.

[3] A. Willig, (2008) "Recent and Emerging Topics in Wireless Industrial Communications: A Selection", IEEE Transactions on Industrial Informatics, Vol. 4, No. 2, pp. 102-122.

[4] A. Cuzzocrea, A. Papadimitriou, D. Katsaros, Y. Manolopoulos, (2012) "Edge between centrality: A novel algorithm for QoS-based topology control over wireless sensor network ”, Elsevier, Journal of network and computer applications, Vol. 35, pp. 1210-1217.

[5] P. Santi, (2005) "Topology Control in Wireless Ad Hoc and Sensor Networks", ACM Computing Surveys, Vol. 37, No. 2, pp. 164-194.

[6] X. Li, Y. Mao, Y. Liang, (2008) “A Survey on Topology Control in Wireless Sensor Networks”, In: 10th Intl. Conf. on Control, Automation, Robotics and Vision, ICARCV, Hanoi, Vietnam, pp. 17-20.

[7] Z.Gengzhong, L. Qiumei, (2010) "A Survey on Topology Control in Wireless Sensor Networks", Second International Conference on Future Networks (ICFN 2010), Sanya, China, January 22-24.

[8] N. Li, J. Hou and L. Sha., (2005) "Design and analysis of an mst-based topology control algorithm", in: Proceedings of the IEEE Infocom, Vol. 4, pp. 1195- 1206.

[9] R. Wattenhofer and A. Zollinger, (2004) "XTC: a practical topology control algorithm for ad-hoc networks". in: Proceedings of the 18th International Parallel and Distributed Processing Symposium, pp. 2-16.

[10] S. Narayanswamy, V. Kawadia, R. S. Sreenivas, P. R. Kumar. (2002) "Power Control in Ad-Hoc networks: Theory, Architecture, Algorithm and Implementation of the COMPOW Protocol". In: Proc. of the European Wireless Conference-Next Generation Wireless Networks: Technologies, Protocols, Services and Applications. Florence, Italy.

[11] A.Venuturumilli and A. Minai., (2006) "Obtaining Robust Wireless Sensor Networks Throuh SelfOrganization of Heterogeneous Connectivity", Proceedings of the 2006 International Conference on Complex Systems (ICCS'06), Boston, MA. 
International Journal of Wireless \& Mobile Networks (IJWMN) Vol. 4, No. 6, December 2012

[12] H. Zhang , J. C. Hou, (2004) "Maintaining sensing coverage and connectivity in large sensor networks," in Proc. of NSF International Workshop on Theoretical and Algorithmic Aspects of Sensor, Ad Hoc Wireless, and Peer-to-Peer networks, Fort Lauderdale, FL, USA,

[13] M. Kubisch, H. Karl, (2003) A.Distributed algorithms for transmission power control in wireless sensor networks.In:YanikomerogluH,ed.Proc.of the IEEE Wireless Communications and Networking Conf.(WCNC).New York:IEEE Press. pp. 16-20.

[14] N. Li, (2004)Topology control in heterogeneous wireless networks:Problems and solutions.In:Proc.of the IEEE Conf.on Computer Communications(INFOCOM).New York:IEEE Press, pp.232-243.

[15] A. Cerpa, (2002) ASCENT:Adaptive self-configuring sensor networks topologies.In:Stojmenovic I,Olariu S,eds.Proc.of the IEEE Conf.on Computer Communications(INFOCOM).New York:IEEE Press.pp. 1278-1287.

[16] F. Ye, (2003) PEAS:A robust energy conserving protocol for long-lived sensor networks. In:Stankovic J,Zhao W, eds. Proc.of the Int'l Conf. on Distributed Computing Systems.Providence:IEEE Press, pp. 28-37.

[17] GL. Xing, XR. Wang,YF. Zhang, CY. Lu, R. Pless, C. Gill, (2005) Integrated coverage and connectivity configuration for energy conservation in sensor networks. ACM Trans. on Sensor Networks ,1(1):36-72.

[18] Z.W. Geem, J.H. Kim, and G.V. Loganathan, (2001) "A new heuristic optimization algorithm: harmony search”, Elsevier Simulation Vol. 76, No. 2, pp. 60-68.

[19] A. Bilal, (2010) "Chaotic harmony search algorithms", Elsevier, Applied Mathematics and Computation , pp. 2687-2699.

[20] G.H. Omran, and M. Mahdavi, (2008) "Global-best harmony search”, Elsevier Applied Mathematics and Computation, pp. 643-656.

[21] J.J. Lotf, S.H. Nazhad, M. Hosseinzadeh, (2011) Applications of learning automata in wireless sensor networks , Proc.of the 5th International Conference on Application of Information and Communication Technologies (AICT), P.P: $1-5$.

[22] M. Mahdavi, M. Fesanghary, and E. Damangir,( 2007) "An improved harmony search algorithm for solving optimization problems", Elsevier, Applied Mathematics and Computation , pp. 1567-1579.

[23] J. Jia, J. Chen, Y. Wen, G. Chang,( 2006) “An extensible core-control routing protocol in large scale ad-hoc networks", in: Proc. of the 6th International Conference on ITS Telecommunications, Chengdu, China, , pp. 955-958.

[24] M. Lu, J. Wu, M. Cardei, M. Li, (2005 ) "Energy-efficient connected coverage of discrete targets in wireless sensor networks", in: Proc. of the International Conference on Computer Networks and Mobile Computing, ICCNMC, Zhangjiajie, China, pp. 43-52.

\section{Authors}

Mohamad Nikravan received the M.S degree in computer engineering from the islamic azad university, Theran north branch, Tehran, Iran, in 2005. He is currently a faculty member at the computer engineering department of islamic azad university, shahr-e-qods branch. His research interests include wireless sensor networks, operating systems and distributed systems.

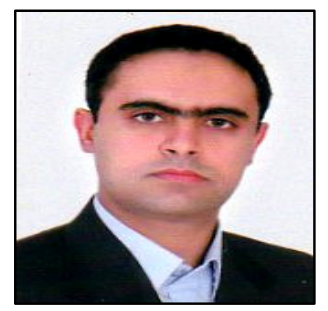


International Journal of Wireless \& Mobile Networks (IJWMN) Vol. 4, No. 6, December 2012

Seyed Mahdi Jameii is a Ph.D. candidate in computer engineering. He is currently a faculty member at the computer engineering department of islamic azad university, shahr-e-qods branch. His research interests include wireless sensor networks, distributed systems and distributed databases.

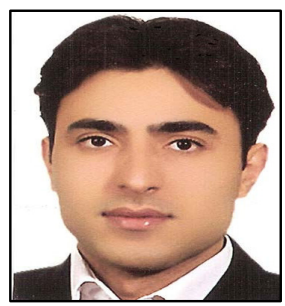

